\title{
Oncolytic adenovirus Ad657 for systemic virotherapy against prostate cancer
}

This article was published in the following Dove Press journal:

Oncolytic Virotherapy

\author{
Tien $\vee$ Nguyen ${ }^{1, *}$ \\ Catherine M Crosby ${ }^{2, *}$ \\ Gregory J Heller ${ }^{3}$ \\ Zachary I Mendel ${ }^{3}$ \\ Mary E Barry' \\ Michael A Barry ${ }^{1,4,5}$ \\ 'Department of Internal Medicine, \\ Division of Infectious Diseases, \\ ${ }^{2} V$ irology and Gene Therapy \\ Graduate Program, Mayo Clinic \\ Graduate School of Biomedical \\ Sciences, ${ }^{3}$ Postbaccalaureate Research \\ Education Program, Mayo Clinic \\ Graduate School of Biomedical \\ Sciences, ${ }^{4}$ Department of Immunology, \\ ${ }^{5}$ Department of Molecular Medicine, \\ Mayo Clinic, Rochester, MN, USA \\ *These authors contributed equally to \\ this work
}

Correspondence: Michael A Barry Mayo Clinic, 200 First Street SW, Rochester, MN 55905, USA

Tel +l 5072669090

Fax + I 50725528 II

Emailmab@mayo.edu
Background: Human species C adenovirus serotype 5 (Ad5) is the archetype oncolytic adenovirus and has been used in the vast majority of preclinical and clinical tests. While Ad5 can be robust, species C Ad6 has lower seroprevalence, side effects, and appears to be more potent as a systemic therapy against a number of tumors than Ad5. Historically, there have only been four species $C$ human adenoviruses: serotypes $1,2,5$, and 6 . More recently a new species $C$ adenovirus, Ad57, was identified. Ad57 is most similar to Ad6 with virtually all variation in their capsid proteins occurring in the hypervariable regions (HVRs) of their hexon proteins. Most adenovirus neutralizing antibodies target the HVRs on adenoviruses. This led us to replace the hexon HVRs in Ad6 with those from Ad57 to create a new virus called Ad657 and explore this novel species C platform's utility as an oncolytic virus.

Methods: The HVR region from Ad57 was synthesized and used to replace the Ad6 HVR region by homologous recombination in bacteria generating a new viral platform that we call Ad657. Replication-competent Ad5, Ad6, and Ad657 were compared in vitro and in vivo for liver damage and oncolytic efficacy against prostate cancers after single intravenous treatment in mice.

Results: Ad5, Ad6, and Ad657 had similar in vitro oncolytic activity against human prostate cancer cells. Ad5 provoked the highest level of liver toxicity after intravenous injection and Ad657 caused the least damage in mice. Previous data demonstrated that Ad6 was superior to Ad5 at killing distant subcutaneous prostate cancer tumors in mouse models after a intravenous injection. Given this, Ad657 was compared to the Ad6 benchmark virus by single intravenous injection into mice bearing subcutaneous human DU145 prostate cancers. Under these conditions, Ad657 first infected the liver and then reached distant tumors. Both Ad6 and Ad657 mediated significant delays in tumor growth and extension of survival with Ad6 mediating higher efficacy. Conclusions: These data suggest that Ad657 may have utility as a local or systemic oncolytic virotherapy for prostate cancers. These data also lay the foundation for serotype-switching with oncolytic species C Ads.

Keywords: adenovirus, oncolytic, seroprevalence, virome, prostate cancer

\section{Introduction}

Adenoviruses (Ads) are nonenveloped DNA viruses that infect a variety of tissues causing respiratory, ocular, and digestive infections. ${ }^{1}$ Most humans are infected by Ads at mucosal surfaces. ${ }^{1}$ Most adenovirus-infected cells die as a consequence of the viral life cycle. Individuals with normal immune systems will reject the viruses after an acute infection. Immunocompromised or stressed individuals can suffer more dangerous, even life-threatening, infections. While adenovirus infections are generally considered acute in terms of disease manifestations, certain human Ads actually become persistent in humans and can be found in T cells in lymphoid and gut tissues. ${ }^{2,3}$ 
Currently, there are in excess of 60 serotypes of Ads that infect humans. Ads were originally segregated into six subtypes A, B, C, D, E, and F based on serologic cross-reactivity and other features. More recently, they have been grouped into six or seven viral "species" with the same letters by DNA sequence comparisons. ${ }^{1}$ These viruses are immunologically and genetically distinct with as much as $40 \%$ genetic divergence across the human adenovirus "virome". 4,5 In general, human Ad serotypes with lower numbers were discovered early in the 1950s and 1960s. These low serotypes were discovered by virtue of the fact that they are already prevalent in humans. ${ }^{1}$ As such, many humans have already been exposed to many of the lower-numbered serotypes and have detectable levels of neutralizing antibodies against them by adulthood. ${ }^{4}$

Early observations that Ads could kill the cervical cancer cell line HeLa prompted human clinical trials in the $1950 \mathrm{~s}$ testing these viruses as oncolytic agents. ${ }^{6,7}$ The use of Ads for this type of oncolytic virotherapy reawakened more recently with the advent of recombinant tools to manipulate their viral genomes (reviewed in Post et $\mathrm{al}^{8}$ and Liu et $\mathrm{al}^{9}$ ). Oncolytic virotherapy utilizes the natural ability of viruses to kill cells and redirects this to kill cancer cells. When an adenovirus kills one cancer cell, it can generate a virus "burst" of as many as 100,000 infectious progeny virions that can kill other cancer cells. This amplification is restricted in immunocompetent hosts by counter amplification of the host's immune cells. Such an antiviral immune response can either quench the wave of expanding oncolytics or may synergize by activating the immune cells against tumor antigens. ${ }^{10}$

The vast majority of recent oncolytic Ad work has focused on the use of human Ad serotype 5 (Ad5). The intense use of Ad5 in preclinical and clinical studies is due in part to the fact that it is one of the most studied viruses, but also because it was the first Ad serotype to be sold as a kit to produce recombinant Ads. While Ad5 is potent against a variety of cancers, it is a low-numbered Ad serotype, and 27\%-100\% of humans are already immune to it. ${ }^{11,12}$ Given this, lower seroprevalent human and non-human Ads that can theoretically avoid preexisting immunity in human cancer patients are currently being tested as vaccines and oncolytics. ${ }^{13-18}$

We evaluated 15 human Ads for oncolytic activity against breast, ovarian, liver, prostate, kidney, and B cell malignancies, emphasizing the testing of viruses that are less seroprevalent than Ad5. ${ }^{17-20}$ In tests against DU145 human prostate tumors, species C Ad6 was more potent after single intratumoral or intravenous (i.v.) injection than species C Ad5 or species B viruses Ad11 and Ad35. ${ }^{17}$ Ad6 was also more effective than Ad5 and Ad11 in immunocompetent Syrian hamsters. ${ }^{20}$

For 50 years, there were only four known species $\mathrm{C}$ human species Ads including Ad1, Ad2, Ad5, and Ad6. ${ }^{21}$ In 2001, a fifth species $\mathrm{C}$ adenovirus was identified as field isolate strain \#16700. ${ }^{22} \# 16700$ was isolated in a fecal sample of a healthy 4-year-old child from Azerbaijan. Virus neutralization testing with antisera against Ad1, 2, 5, and 6 demonstrated high

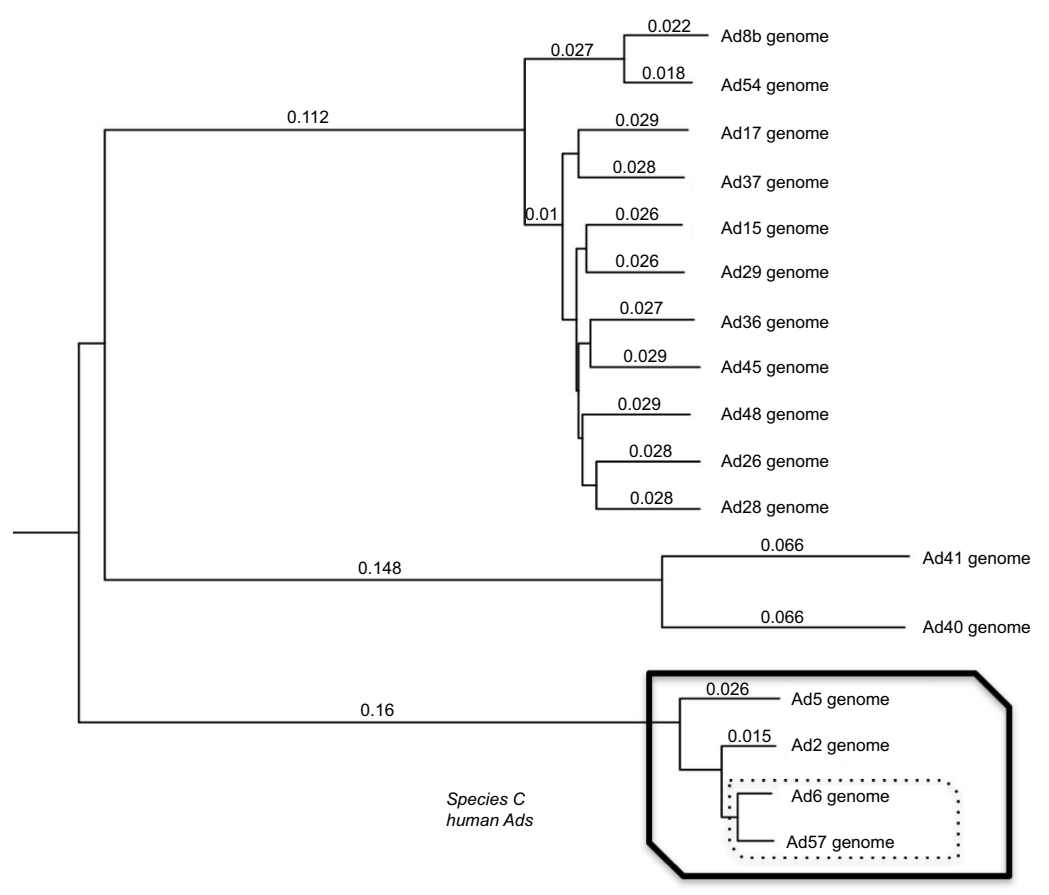

Figure I Phylogenetic tree of whole genome sequences of human adenovirus serotypes. 
levels of neutralization (reciprocal titers of 500-16,000) when each antisera was used against its cognate virus. ${ }^{22}$ In contrast, anti-Ad1, 2, and 5 antibodies have weak cross-reactivity against \#16700 (reciprocal titers of 32-64). Anti-Ad6 sera demonstrated higher cross-reactivity against \#16700, but neutralization required 10 -fold higher concentrations of sera to neutralize \#16700 when compared with Ad6 itself.
Subsequent sequence comparisons confirmed $\# 16700$ as a novel species $\mathrm{C}$ adenovirus and renamed it as Ad57 (Figure 1). ${ }^{23}$ For example, alignment of selected full Ad genomes produces a phylogenetic tree that clusters Ad57 with other species $\mathrm{C}$ viruses with most homology with Ad6.

Ad57 appears nearly identical to Ad6 with sequence divergence in hexon hypervariable regions (HVRs) and in

A

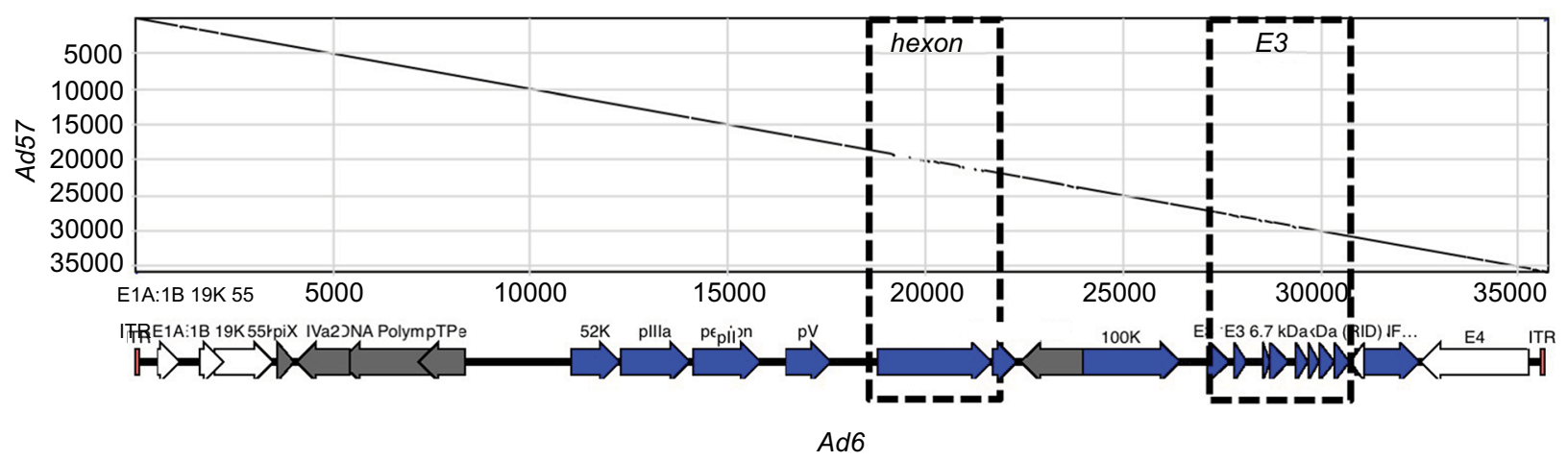

B

HVR1

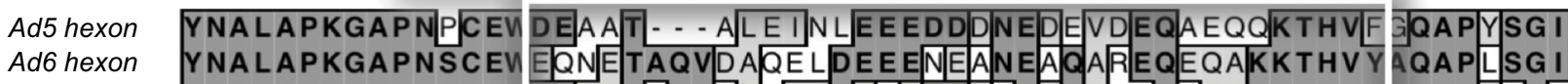

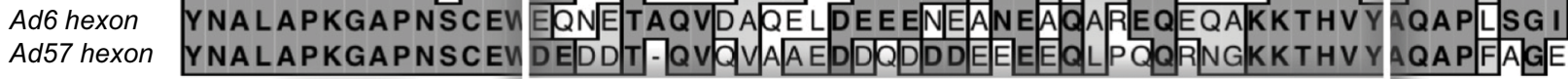
HVR2 HWIR3

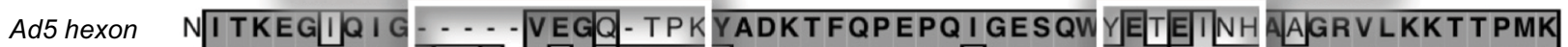
Ad6 hexon K I TKEGLQ I G TADATVAGAGKE I FADKTFQP EPQV]GESQWNE ADATA AGGRVLKKT T PMK

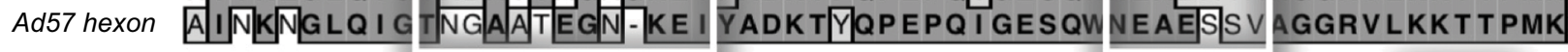
HVR4

HVRT5

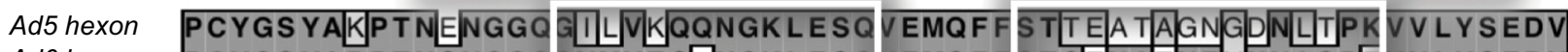
Ad6 hexon PCYGS YARPTNSNGGQGVMVEQ-NGKLESQVEMQFFS TSTNATNEVINN I QPTVVLYSEDV Ad57 hexon PCYGSYARPTNSNGGQGVMVEQ-NGKLESQVEMQFFSTSVNAMNEANA I QPK LIVLYSEDV HVR6

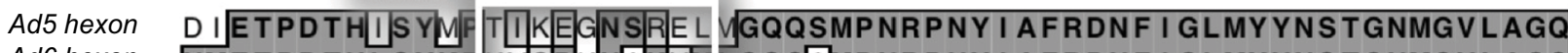

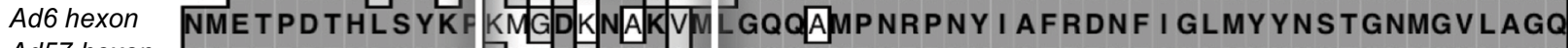
Ad57 hexon NMETPDTHLSYKF GKSDDNSKAMLGQQSMPNRPNY I AFRDNF I GL MY YNSTGNMGVLAGQ

Ad5 hexon Ad6 hexon Ad57 hexon

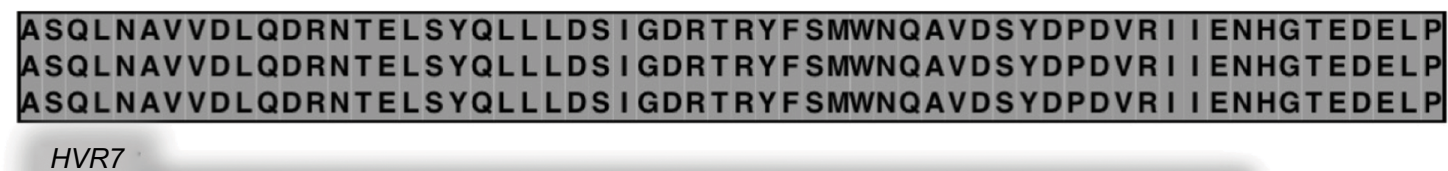
HVR7

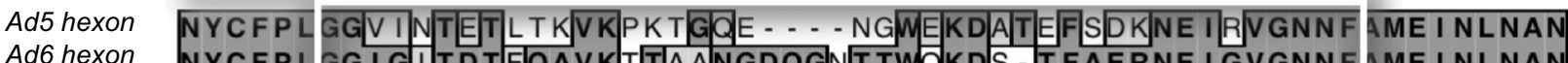

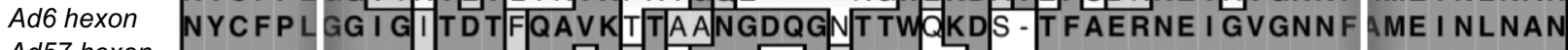

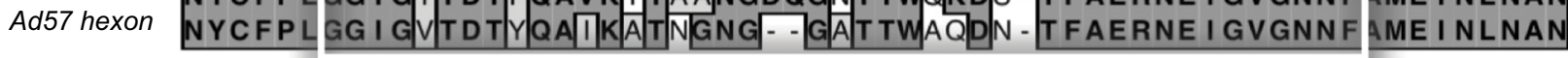

Ad5 hexon LWRNF LYSN I ALYLPDKLKYSPSNVKI SDNPNTYDYMNKRVVAPGLVDCY INLGARWSLD Ad6 hexon LWRNF LYSN I ALYLPDKLKYNPTNVE I SDNPNTYDYMNKRV VAPGLVDCY I NLGARWSLE Ad57 hexon LWRNFLYSN I ALYLPDKLKYNPTNVE I SDNPNTYDYMNKRV VAPGLVDCY INLGARWSLD

Figure 2 Alignment of $A d 5,6$, and 57 showing variation in hexon and $E 3$ regions.

Notes: (A) A Pustell DNA alignment of the genomes of Ad6 and Ad57. Boxes indicate hexon and E3 regions where variation is highest between the two viruses. (B) ClustalW amino acid alignment of the hypervariable region in hexon proteins from Ad5, Ad6, and Ad57. Alignments were performed on MacVector. 
E3 immune evasion genes ${ }^{22,23}$ (Figure 2). Other exposed viral capsid proteins including fiber, penton base, IIIa, and IX are virtually identical between Ad6 and 57. This and the neutralization data are consistent with the fact that most adenovirus-neutralizing antibodies target the HVRs on Ads. ${ }^{24,25}$ The low cross-reactivity between Ad6 antisera and Ad57 is thought to be due to antibodies that may target their common fiber protein. ${ }^{22}$

Ad57 is fortuitously most similar to our most effective oncolytic adenovirus, Ad6. Given this, in this work, we have explored the utility of Ad57 as an oncolytic against human prostate cancer. Given that Ad6 and 57 differ only in their hexon proteins, we replaced the Ad6 HVRs with those from Ad57 to generate a new species $\mathrm{C}$ oncolytic virus called Ad657 for this testing. We tested Ad657 and Ad6 as systemic oncolytic therapies by single i.v. injection in nude mice bearing human prostate cancer tumors. We evaluated the liver and tumor tropism of this virus in mouse models of prostate cancer.

\section{Materials and methods Cell lines}

DU145 human prostate carcinoma cells were purchased from American Type Culture Collection (ATCC; Manassas, VA, USA) and verified to be specific pathogen free by IMPACT testing by RADIL. 293 cells were obtained from Microbix, Toronto, Ontario, Canada. Cells were maintained in DMEM with 10\% FBS (Invitrogen, Grand Island, NY, USA).

\section{Viruses}

The genome of Ad6 Tonsil 99 strain (ATCC VR-1083) was cloned previously. ${ }^{26}$ A cassette corresponding to the Ad57 hexon between a natural ApaI and SacI sites was synthesized by Genscript. This fragment was cloned into the shuttle plasmid pUC57-Ad6 Hexon-FZF containing the Ad6 pVI and hexon genes with a FRT-Zeocin resistance gene-FRT cassette between them for homologous recombination in bacteria as in Campos and Barry ${ }^{27}$ and Khare et al. ${ }^{28}$ The Ad6 ApaI-SacI fragment was replaced with the Ad57 fragment generating the plasmid pUC57-Ad6/57 Hexon-FZF. This was recombined into the Ad6 genome by red recombination. ${ }^{27}$ Viruses were rescued by transfection into 293 cells and produced from a 10 plate CellStack (Corning Life Sciences, Lowell, MA, USA). Viruses expressing a green fluorescent protein-luciferase (GFP-Luc) fusion protein have a CMV-GFP-Luc expression cassette inserted between fiber and E4 and an E3 deletion to make space for this insertion. ${ }^{29}$ Viruses were purified on two $\mathrm{CsCl}$ gradients, and viral particle (vp) numbers were calculated by OD260.

\section{In vitro oncolytic activity}

Cells were treated at the indicated multiplicities of infection (MOI) in terms of vp/cell in DMEM with 5\% FBS and antibiotic-antimycotic (Invitrogen, Grand Island, NY, USA). Five days later, media was removed and the cells were treated with crystal violet $(0.05 \%$ crystal violet, $3.7 \%$ formaldehyde, in phosphate-buffered saline; Invitrogen, Grand Island, NY, USA) for 10 minutes. The cells were washed twice with PBS and then incubated overnight at $37^{\circ} \mathrm{C}$ in $0.1 \%$ sodium dodecyl sulfate in PBS to solubilize the crystal violet. Crystal violet absorbance was measured at OD595 on a Beckman Coulter DTX 880 plate reader. Cell viability (\%) was calculated by dividing the OD of the samples by the mean OD of untreated control cells on the same 96-well plate and multiplying this number by 100 .

\section{Animals}

Animals were housed in the Mayo Clinic Animal Facility under Association for Assessment and Accreditation of Laboratory Animal Care guidelines. The studies were approved by the Mayo Clinic Animal Use and Care Committee under the provisions of the Animal Welfare Act, PHS Animal Welfare Policy. Subcutaneous tumors were initiated in 4-week-old nude mice (Harlan Sprague Dawley, Indianapolis, IN, USA) by injecting subcutaneously (s.c.) with $1 \times 10^{7} \mathrm{DU} 145$ cells in $100 \mu \mathrm{L}$ of DMEM $/ 50 \%$ Matrigel (BD Biosciences, San Jose, CA, USA). Tumor volumes were calculated using the equation width ${ }^{2} \times$ length $\times 1 / 2$. When tumors reached $\sim 200 \mu \mathrm{L}$ in volume, mice were distributed into different groups and were treated by a single i.v. injection tail vein. Animals were euthanized when the tumor volume reached $2000 \mu \mathrm{L}$ or if animals were moribund, in distress, or if the skin ruptured over the tumor.

\section{Blood alanine aminotransferase (ALT) measurement}

Groups of six C57BL/6 mice were injected i.v. with $10^{11} \mathrm{vp}$ of Ad5, Ad6, or Ad657 by tail vein and blood was collected 3 days later for ALT measurement using ALT Activity Assay (Sigma-Aldrich, St. Louis, MO, USA).

\section{Statistical analysis}

Statistical analysis was performed with Prism (Graphpad) by repeated measures ANOVA or one-way ANOVA followed by Tukey's HSD test. Kaplan-Meier survival curves were plotted and compared by log rank test. 


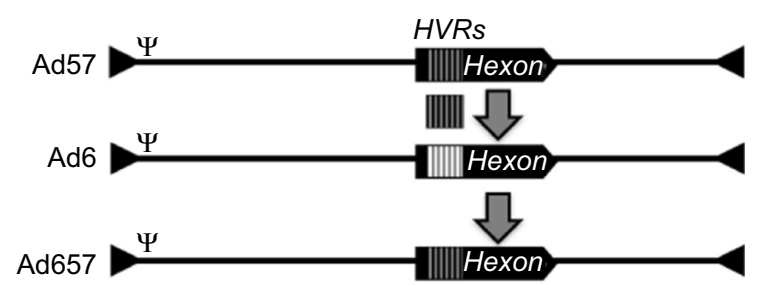

Figure 3 Cartoon of the construction of Ad657 by replacement of the Ad6 HVRs with Ad57 HVRs.

Abbreviation: HVRs, hypervariable regions.

A

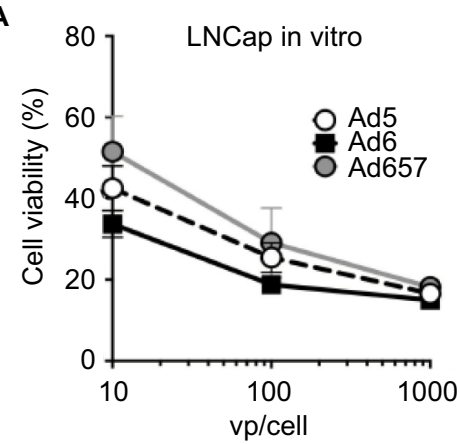

B

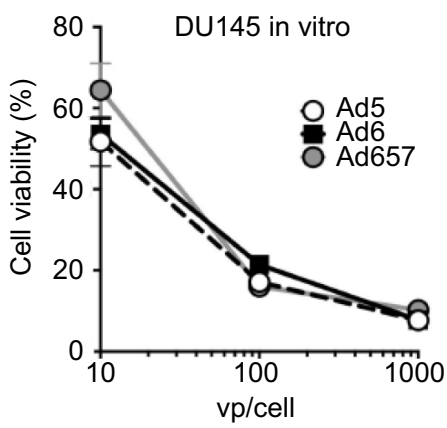

Figure 4 In vitro oncolytic activity.

Notes: LNCaP and DUI 45 cells were treated with the indicated viruses with the indicated vp/cell for 5 days. The cells were stained with crystal violet and cell viability was measured by OD595. Cell viability (\%) was calculated by dividing the OD of the samples by the mean OD of untreated control cells on the same 96-well plate and multiplying this number by 100. (A) LNCaP cell killing. (B) DUI45 killing. Abbreviation: vp, viral particle.

\section{Results}

\section{Construction of new Ad657 virus}

We previously cloned the genome of human Ad6 Tonsil 99 strain. ${ }^{26}$ The capsomer genes of Ad57 are nearly identical to Ad6 with the exception of their hexon HVRs (Figures 1 and 2) ${ }^{22,23}$ To generate a mimic of Ad57, a cassette corresponding to the Ad57 hexon HVRs was recombined into the wild-type Ad6 genome (Figure 3). This virus was rescued and produced in 293 cells and purified on $\mathrm{CsCl}$ gradients. Given that the base viral genome is Ad6, hereafter, we refer to these hexon chimeric viruses as Ad657.

\section{In vitro oncolytic activity}

LNCaP and DU145 human prostate cancer cells were treated with Ad5, Ad6, and Ad657 at varied MOI in vp/cell. Five days later, the viability of the cells was estimated by staining them with crystal violet. Under these conditions, the three viruses mediated similar levels of LNCaP and DU145 cell killing (Figure 4).

\section{Liver damage by Ad5, Ad6, and Ad657}

Systemic oncolytic therapy may require i.v. injections of viruses. While this can distribute oncolytic Ads to distant tumors, the vast majority of i.v. injected virus becomes trapped in the liver (reviewed in Barry et $\mathrm{l}^{130}$ ). This can cause profound liver damage and even death in a serotypedependent manner. For example, in immunocompetent mice, Ad5 causes substantially higher ALT levels in the blood and significantly higher morbidity than Ad6. ${ }^{17,31}$

To compare liver damage by Ad5, Ad6, and Ad657, a high dose of $10^{11} \mathrm{vp}$ of each virus were injected by tail vein into immunocompetent C57BL/6 mice. Consistent with previous findings, ${ }^{31}$ Ad5-injected animals became moribund within 2 days and had to be euthanized (Figure 5A). Survival for Ad5 and Ad6 was significantly lower when compared with PBS ( $p=0.0001$ and 0.0009 , respectively, by log-rank analysis). Survival for Ad657 was also reduced when compared with PBS ( $p=0.0578$ ). Survival after exposure to Ad6 or Ad657 was significantly better than in Ad5-treated mice ( $p=0.0001$ and 0.0001, respectively). Ad6 and Ad657 survival were not statistically different $(p=0.248)$.

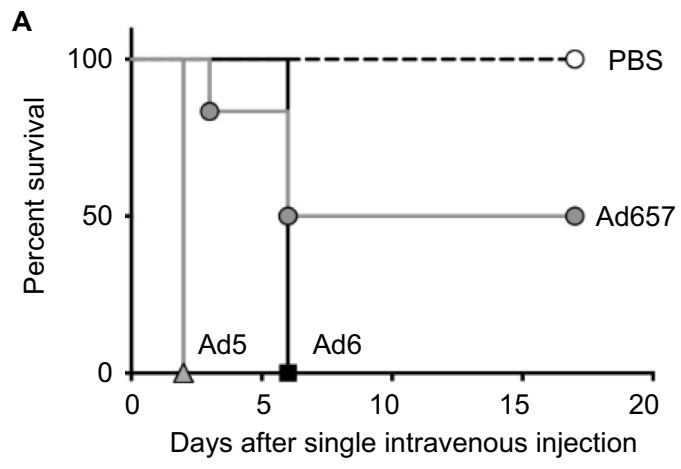

B

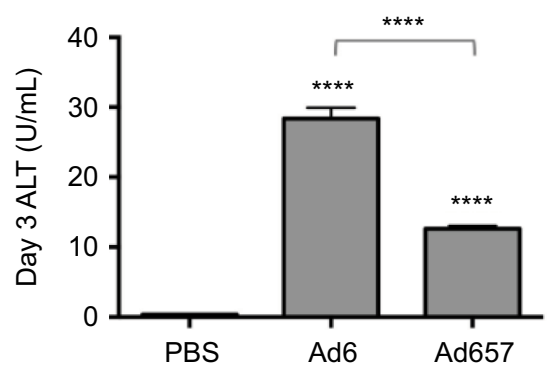

Figure 5 Effects of oncolytic Ads on liver damage.

Notes: C57BL/6 mice ( $\mathrm{n}=6$ per group) were injected with $10^{11}$ vp of each virus by tail vein. (A) Kaplan-Meier survival. (B) Blood was drawn for ALT measurements 3 days after injection (****p<0.001 by ANOVA).

Abbreviations: ALT, alanine aminotransferase; vp, viral particle. 
ALT was measured in the blood 3 days after injection in surviving Ad6 and Ad657 animals. Ad5-treated animals were not tested, since most of the group needed to be sacrificed. This assay showed that Ad6 provoked relatively low levels of liver damage in terms of liver ALT enzyme release in the blood (Figure 5B) consistent with previous results. ${ }^{17,31}$ Both Ad6 and Ad657 groups had low, but significant, ALT levels when compared with PBS-treated mice $(p<0.001$ by oneway ANOVA with Tukey's multiple comparison test for both viruses). Ad657 had lower ALT levels than Ad6 ( $p<0.001$ by ANOVA). This is consistent with higher levels of Ad6 infection in the liver than Ad657 after i.v. injection of luciferase expressing viruses (Figure S1). One Ad657 animal was lost following bleeding on day 3. By 6 days, most of the Ad6 animals became moribund (Figure $5 \mathrm{~A}$ ). In contrast, $50 \%$ of Ad657 animals survived beyond 2 weeks of the treatment.

\section{Comparison of the oncolytic activity of Ad6 and Ad657 against human DUI45 prostate tumors}

We previously showed that species C Ad6 was more potent after single intratumoral or i.v. injection than Ad5 or species B Ad11 and Ad35 against DU145 prostate carcinoma tumors. ${ }^{17}$ Ad6 expressing luciferase first infects the liver and then expands in distant subcutaneous DU145 prostate tumors after i.v.injection. ${ }^{31}$

To compare Ad6 and Ad657 against this tumor type, nude mice were engrafted s.c. with DU145 cells. Animals were distributed into groups with similar tumor sizes averaging $200 \mu \mathrm{L}$ and groups of nine mice were treated a single time by the i.v. route with a dose of $3 \times 10^{10} \mathrm{vp}$ of Ad6 or Ad657 (Figure 6).

This single i.v. injection of Ad6 and Ad657 reduced tumor sizes when compared with PBS-injected control animals. Tumors were significantly smaller in the Ad6 group within 7 days when compared with the PBS group ( $p<0.05$ by two-way ANOVA with Tukey's multiple comparison test). Tumors in the Ad657 group were significantly different from those in the PBS group by day 14 ( $p<0.01$ by ANOVA). Both Ad6 and Ad657 maintained significant differences with PBS through day 38 ( $p<0.0001$ by two-way ANOVA). This comparison ended on day 38 when the first animal in the PBS group had to be sacrificed since later comparison would be skewed due to the change in animal numbers. Tumor sizes in the Ad6 and Ad657 groups were not significantly different until day 38, when Ad657 had a significantly higher tumor volume $(p<0.05)$ by two-way ANOVA (Figure 6A). This difference between Ad6 and Ad657 tumor sizes persisted until day 52

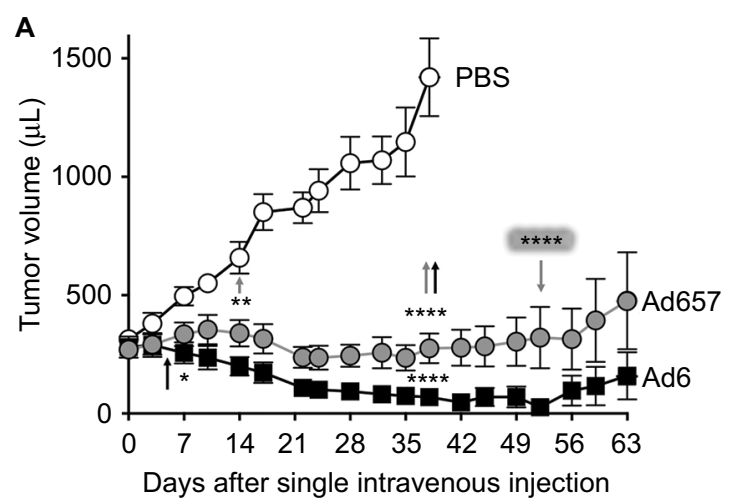

B

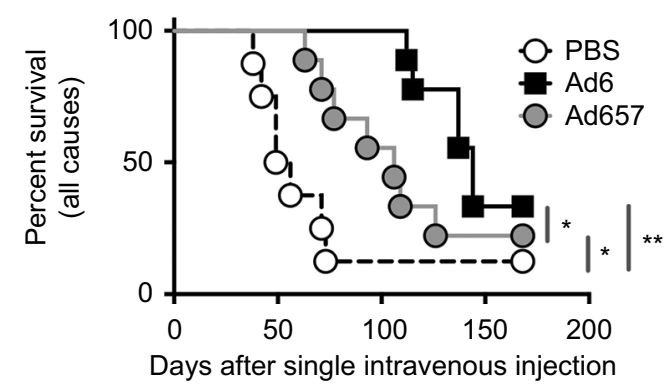

Figure 6 Anticancer activity of Ad6 and Ad657 in DU145 tumor xenografts in nude mice after single i.v. administration.

Notes: Nude mice ( $\mathrm{n}=9$ per group) bearing established DUI45 tumors were injected i.v. with a single dose of $3 \times 10^{10} \mathrm{vp}$ of the indicated viruses or with PBS. (A) Effect of a single i.v. injection on tumor growth. Tumor dimensions were measured with calipers and tumor volume was calculated as width ${ }^{2} \times l$ length $\times I / 2$. The data are shown as mean \pm SE. $* p<0.05, * * * * p=0.000$ I by ANOVA or by $T$-test as described in the text. Black asterisks with a black arrow pointing up indicate the statistical difference between the Ad6 group and the PBS group on a selected day described in the text. Gray asterisks and an arrow pointing up indicate differences between the Ad657 group and the PBS group on the indicated day. The shadowed white asterisks with a gray arrow pointing down indicates the statistical difference between the Ad6 and Ad657 groups on the indicated day. (B) Effect of a single i.v. injection on survival. Animals were euthanized when the tumor volume reached $2000 \mu \mathrm{L}$ or when other sacrifice criteria were met (e.g., ulceration) and Kaplan-Meier survival curves were plotted $\left({ }^{*} p<0.05,{ }^{* *} p<0.01\right.$ by log-rank analysis).

Abbreviation: i.v., intravenous.

( $\mathrm{p}=0.04$ by $T$-test), and then the tumors were not significantly different after this time.

When survival due to all causes was assessed, both Ad6 and Ad657 significantly extended survival when compared with PBS-treated animals (Figure 6B, $p<0.01$ and 0.05, respectively, by log-rank analysis). Ad6 survival due to all causes was significantly better than Ad657 $(p<0.05)$. However, this was an artifact of survival attributed to all because three of the Ad657 animals had to be sacrificed per Institutional Animal Care and Use Committee (IACUC) guidelines due to the formation of ulcers on the skin over the tumor rather than due to excess tumor size. In some cases, ulceration is actually associated with effective tumor control. Like Ad6, ${ }^{31}$ Ad657 expressing GFP-luciferase produced significant luciferase activity in distant DU145 subcutaneous tumors after a single i.v. injection (Figure S2). This suggests 
that both Ad6 and Ad657 can mediate oncolytic effects in prostate tumors after a single systemic treatment.

\section{Discussion}

Human species C Ad5 is a potent oncolytic virus for many solid tumors. ${ }^{17-20}$ Its lower seroprevalence species $C$ cousin, Ad6 is more effective in mice and hamsters than Ad5, particularly by the i.v. route. ${ }^{17,20}$ This work was directed at providing a new species $C$ variant's hexon hypervariability to the oncolytic Ad palette.

For 50 years, there were only four species $C$ human species Ads including Ad1, Ad2, Ad5, and Ad6. ${ }^{21} \mathrm{Ad} 57$ is a new species $C$ adenovirus most similar to lower seroprevalence Ad6 with most of its sequence divergence in its surface proteins focused on hexon HVRs (Figure 2). ${ }^{22,23}$ Ad57 has very low serologic cross-reactivity to antibodies generated against Ad1, 2, and 5.22 Anti-Ad6 sera had higher cross-reactivity against Ad57, but this required 10 times more sera to neutralize Ad57 than Ad6 itself. Given that most Ad neutralization is directed at hexon HVRs, we constructed Ad657 to evade Ad6 HVR-directed antibodies on the potent Ad6 genomic platform.

Previous comparisons of Ad5 and Ad6 against DU145 prostate tumors in nude mice showed that Ad6 mediated better tumor size control and survival than species C Ad5 or species B Ad1 1 and Ad35 after i.v. treatment. ${ }^{17}$ Interestingly, subsequent comparisons of more human Ads against MDAMB-468 human breast cancer and SKOV3 human ovarian tumors in nude mice showed that Ad5 and Ad6 had similar efficacy, but that other Ads were more potent. ${ }^{20}$ Species B Ad1 1 and species D Ad26 controlled breast tumor size best, whereas Ad1 1 was best against ovarian cancer. ${ }^{20}$ In contrast, in B cell cancers, species D Ads are superior to most other Ads including Ad5, Ad6, Ad11, and Ad35. ${ }^{18,19}$ When Ad5, Ad6, and Ad11 were tested in immunocompetent hamsters against HaK kidney tumors, only Ad6 mediated significant reductions in tumor growth. ${ }^{20}$ This suggests that different Ads indeed have better utility against different cancer types. Of all Ads tested, unmodified species $\mathrm{C}$ Ads appear to have a particular "hunger" for prostate cancer.

In this study, species C Ad6 and Ad657 were both effective against distant subcutaneous DU145 tumors after systemic delivery. It should be noted that this efficacy is mediated by a single i.v. dose rather than by multiple treatments. Under these conditions, both Ad6 and Ad657 repressed aggressive DU145 tumor growth over long periods of time after this single treatment. Ad6 had slightly better tumor control than Ad657 over time, but both mediated similar control of tumor size over time.
Survival due to all causes was better in Ad6-treated animals, but this was due in part to a higher rate of ulceration in the skin above the tumor sites in Ad657 animals. These animals were sacrificed per IACUC guidelines skewing the survival curve. In some cases, ulceration is actually associated with more effective tumor control, so it is possible that the two species $\mathrm{C}$ oncolytic viruses may have similar activity in models less prone to ulceration.

Liver sequestration is a considerable problem for virtually any oncolytic virus if it is used as an i.v. systemic therapy or if viremia occurs after intratumoral injection. If said virus infects hepatocytes and kills them, this will result in liver damage at low doses and death at higher doses. When we compared liver damage in immunocompetent mice by Ad5, Ad6, and Ad657, both Ad6 and Ad657 provoked lower damage than Ad5. Under these conditions, Ad657's liver damage was significantly lower than that mediated by Ad6. It could well be that liver damage may be higher in immunodeficient mice, although this was not tested here.

Ad5 was originally reported to have seroprevalence of $27 \%$ in Texas. ${ }^{32}$ In contrast, Ad6 seroprevalence was only $3 \% .{ }^{32}$ Other human Ads including Ad26 and Ad35 have even lower seroprevalence than Ad6, ${ }^{12,33}$ but have less efficacy against prostate cancer after systemic injection than Ad6. ${ }^{17}$ Non-human Ads like chimpanzee ChAd3 have lower seroprevalence than human Ads, but this is not as low as first expected. For example, the ranked order of preexisting antibodies against $\mathrm{Ad} 5, \mathrm{Ad} 6$, and $\mathrm{ChAd} 3$ are 38\%, 22\%, and $10 \%$, respectively. ${ }^{34}$ Recent comparison of Ad6 and ChAd3 as hepatitis $\mathrm{C}$ vaccines in humans showed that both generated robust immune responses. ${ }^{34}$ This suggests that Ad6 can have utility against prostate cancer in humans. While it is as yet uncertain what the seroprevalence of Ad57 is in humans, the fact that it has been isolated only once in humans ${ }^{22}$ suggests that it will be even less common than Ad6. Antibodies against Ad1, 2, and 5 cross-react weakly against Ad6 suggesting that preexisting immunity against these common viruses will not impede the use of Ad6 in prostate cancer patients. This is in marked contrast to $\mathrm{Ad} 5$ that has been used in the vast majority of human trials.

Anti-Ad6 sera have some cross-reactivity to Ad57, but both viruses avoid each other's antibodies 10 -fold better than they avoid their own antibodies. ${ }^{22}$ This suggests that Ad6 and Ad657 could both be used for serial therapy in prostate cancer patients.

Of all Ads, unmodified species C Ads appear to have a particularly higher efficacy against prostate cancer. Species C Ad6 and Ad657 have lower prevalence than archetype Ad5. 
This suggests that Ad6 and Ad657 may be useful as single or dual oncolytic or vaccine platforms against prostate cancer in humans.

\section{Disclosure}

The authors report no conflicts of interest in this work.

\section{References}

1. Davison AJ, Benko M, Harrach B. Genetic content and evolution of adenoviruses. J Gen Virol. 2003;84(Pt 11):2895-2908.

2. Roy S, Vandenberghe LH, Kryazhimskiy S, et al. Isolation and characterization of adenoviruses persistently shed from the gastrointestinal tract of non-human primates. PLoS Pathogens. 2009;5(7):e1000503.

3. Garnett CT, Talekar G, Mahr JA, et al. Latent species C adenoviruses in human tonsil tissues. J Virol. 2009;83(6):2417-2428.

4. Barry MA, Weaver EA, Chen CY. Mining the adenovirus "Virome" for systemic oncolytics. Curr Pharm Biotechnol. 2012;13(9):1804-1808.

5. Weaver EA, Hillestad ML, Khare R, Palmer D, Ng P, Barry MA. Characterization of species $\mathrm{C}$ human adenovirus serotype 6 (Ad6). Virology. 2011;412(1):19-27.

6. Pereira HG, Kelly B. Dose-response curves of toxic and infective actions of adenovirus in HeLa cell cultures. J Gen Microbiol. 1957;17(2):517-524.

7. Huebner RJ, Rowe WP, Schatten WE, Smith RR, Thomas LB. Studies on the use of viruses in the treatment of carcinoma of the cervix. Cancer. 1956;9(6):1211-1218.

8. Post DE, Khuri FR, Simons JW, Van Meir EG. Replicative oncolytic adenoviruses in multimodal cancer regimens. Hum Gene Ther. 2003;14(10):933-946.

9. Liu TC, Galanis E, Kirn D. Clinical trial results with oncolytic virotherapy: a century of promise, a decade of progress. Nat Clin Pract Oncol. 2007;4(2):101-117.

10. Prestwich RJ, Errington F, Diaz RM, et al. The case of oncolytic viruses versus the immune system: waiting on the judgment of Solomon. Hum Gene Ther. 2009;20(10):1119-1132.

11. Piedra PA, Poveda GA, Ramsey B, McCoy K, Hiatt PW. Incidence and prevalence of neutralizing antibodies to the common adenoviruses in children with cystic fibrosis: implication for gene therapy with adenovirus vectors. Pediatrics. 1998;101(6):1013-1019.

12. Abbink P, Lemckert AA, Ewald BA, et al. Comparative seroprevalence and immunogenicity of six rare serotype recombinant adenovirus vaccine vectors from subgroups B and D. J Virol. 2007;81(9):4654-4663.

13. Hemminki A, Kanerva A, Kremer EJ, et al. A canine conditionally replicating adenovirus for evaluating oncolytic virotherapy in a syngeneic animal model. Mol Ther. 2003;7(2):163-173.

14. Hoffmann D, Bayer W, Heim A, Potthoff A, Nettelbeck DM, Wildner O. Evaluation of twenty-one human adenovirus types and one infectivityenhanced adenovirus for the treatment of malignant melanoma. J Invest Dermatol. 2008;128(4):988-998.

15. Hoffmann D, Heim A, Nettelbeck DM, Steinstraesser L, Wildner O. Evaluation of twenty human adenoviral types and one infectivityenhanced adenovirus for the therapy of soft tissue sarcoma. Hum Gene Ther. 2007;18(1):51-62.
16. Strauss R, Sova P, Liu Y, et al. Epithelial phenotype confers resistance of ovarian cancer cells to oncolytic adenoviruses. Cancer Res. 2009;69(12):5115-5125.

17. Shashkova EV, May SM, Barry MA. Characterization of human adenovirus serotypes 5, 6, 11, and 35 as anticancer agents. Virology. 2009;394(2):311-120.

18. Senac JS, Doronin K, Russell SJ, Jelinek DF, Greipp PR, Barry MA. Infection and killing of multiple myeloma by adenoviruses. Hum Gene Ther. 2010;21(2):179-190.

19. Chen CY, Senac JS, Weaver EA, et al. Species D adenoviruses as oncolytics against B-cell cancers. Clin Cancer Res. 2011;17(21)6712-6722.

20. Chen CY, Weaver EA, Khare R, May SM, Barry MA. Mining the adenovirus virome for oncolytics against multiple solid tumor types. Cancer Gene Ther. 2011;18(10):744-750.

21. Weaver EA, Hillestad ML, Khare R, Palmer D, Ng P, Barry MA. Characterization of species $C$ human adenovirus serotype 6 (Ad6). Virology. 2011;412(1):19-27.

22. Lukashev AN, Ivanova OE, Eremeeva TP, Iggo RD. Evidence of frequent recombination among human adenoviruses. $J$ Gen Virol. 2008;89:380-388

23. Walsh MP, Seto J, Liu EB, et al. Computational analysis of two species $\mathrm{C}$ human adenoviruses provides evidence of a novel virus. $J$ Clin Microbiol. 2011;49(10):3482-3490.

24. Pichla-Gollon SL, Drinker M, Zhou X, et al. Structure-based identification of a major neutralizing site in an adenovirus hexon. $J$ Virol. 2007;81(4):1680-1689.

25. Sumida SM, Truitt DM, Lemckert AA, et al. Neutralizing antibodies to adenovirus serotype 5 vaccine vectors are directed primarily against the adenovirus hexon protein. J Immunol. 2005;174(11):7179-7185.

26. Weaver EA, Barry MA. Low seroprevalent species D adenovirus vectors as influenza vaccines. PLoS One. 2013;8(8):e73313.

27. Campos SK, Barry MA. Rapid construction of capsid-modified adenoviral vectors through bacteriophage lambda red recombination. Hum Gene Ther. 2004;15(11):1125-1130.

28. Khare R, Reddy VS, Nemerow GR, Barry MA. Identification of adenovirus serotype 5 hexon regions that interact with scavenger receptors. J Virol. 2012;86(4):2293-2301.

29. Crosby CM, Barry MA. IIIa deleted adenovirus as a single-cycle genome replicating vector. Virology. 2014;462-463:158-165.

30. Barry MA, Hofherr SE, Chen CY, Senac JS, Hillestad ML, Shashkova EV. Systemic delivery of therapeutic viruses. Curr Opin Mol Ther. 2009;11(4):411-420.

31. Nguyen TV, Heller GJ, Barry ME, Crosby CM, Turner MA, Barry MA. Evaluation of polymer shielding for adenovirus serotype 6 (Ad6) for systemic virotherapy against human prostate cancers. Mol Ther Oncolytics. 2016;3:15021.

32. Munoz FM, Piedra PA, Demmler GJ. Disseminated adenovirus disease in immunocompromised and immunocompetent children. Clin Infect Dis. 1998;27(5):1194-1200.

33. Thorner AR, Vogels R, Kaspers J, et al. Age dependence of adenovirusspecific neutralizing antibody titers in individuals from sub-Saharan Africa. J Clin Microbiol. 2006;44(10):3781-3783.

34. Colloca S, Barnes E, Folgori A, et al. Vaccine vectors derived from a large collection of simian adenoviruses induce potent cellular immunity across multiple species. Sci Transl Med. 2012;4(115):115ra112. 


\section{Supplementary materials}

Ad6-GL

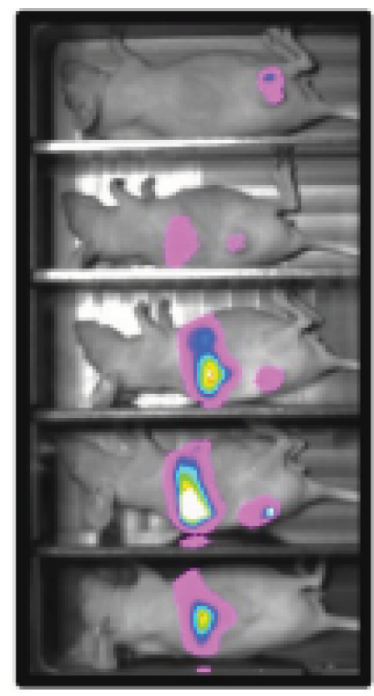

Ad657-GL

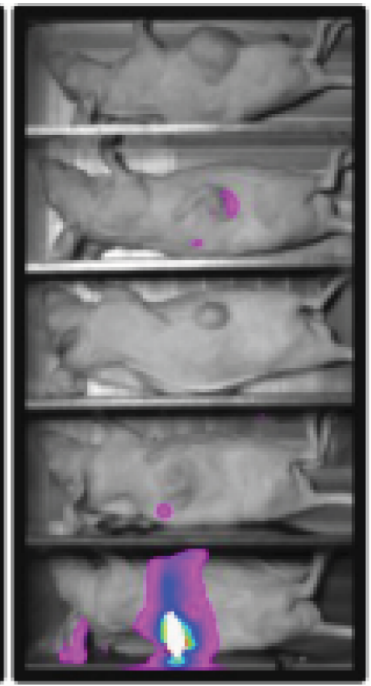

Figure SI Luciferase imaging nude mice.

Notes: Four days after single i.v. injection of $3 \times 10^{10}$ vp of Ad6 and Ad657-GFP-Luc.

Abbreviations: i.v., intravenous; vp, viral particle.

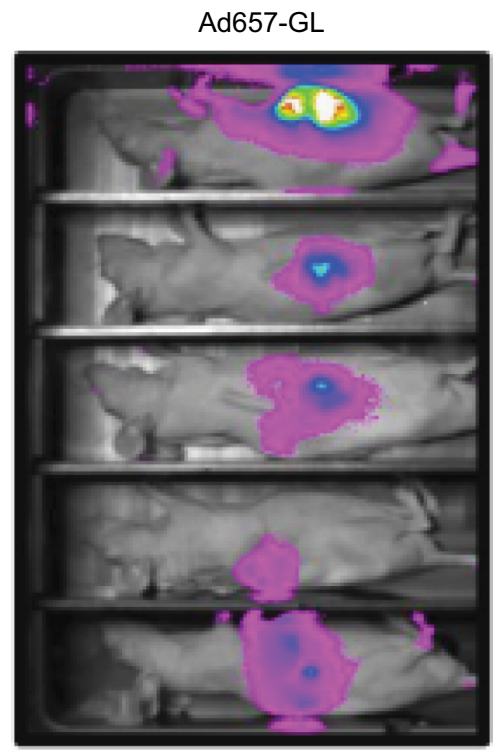

Figure S2 Luciferase imaging nude mice.

Notes: Fourteen days after single i.v. injection of $3 \times 10^{10} \mathrm{vp}$ of Ad657-GFP-Luc.

Abbreviations: i.v., intravenous; vp, viral particle.

Oncolytic Virotherapy

\section{Publish your work in this journal}

Oncolytic Virotherapy is an international, peer-reviewed, open access online journal publishing original research, study protocols, reviews, editorials and commentaries on all aspects of oncolytic virology, namely the application of oncolytic viruses for the treatment of cancer. Specific topics in the journal include: Rationale and theoretical aspects of oncolytic virotherapy including in vitro, in vivo and mathematical

\section{Dovepress}

modeling; and practical application and problem solving in the clinic including identification of potential responders through biomarkers and genetic profiling. The manuscript management system is completely online and includes a very quick and fair peer-review system, which is all easy to use. Visit http://www.dovepress.com/ testimonials.php to read real quotes from published authors. 